\title{
A Note on the Standard Embedding on Half-Flat Manifolds
}

\author{
Tibra Ali* and Gerald B. Cleaver ${ }^{\dagger}$ \\ Physics Department \\ Baylor University \\ One Bear Place \# 97316 \\ Waco, Texas 76798 \\ U.S.A
}

\begin{abstract}
It is argued that the ten dimensional solution that corresponds to the compactification of $E_{8} \times E_{8}$ heterotic string theory on a half-flat manifold is the product spacetime $\mathbb{R}^{1,2} \times \mathcal{Z}_{7}$ where $\mathcal{Z}_{7}$ is a generalized cylinder with $G_{2}$ riemannian holonomy. Standard embedding on $\mathcal{Z}_{7}$ then implies an embedding on the half-flat manifold which involves the torsionful connection rather than the LeviCivita connection. This leads to the breakdown of $E_{8} \times E_{8}$ to $E_{6} \times E_{8}$, as in the case of the standard embedding on Calabi-Yau manifolds, which agrees with the result derived recently by Gurrieri, Lukas and Micu [1] using a different approach. Green-Schwarz anomaly cancellation is then implemented via the torsionful connection on halfflat manifolds.
\end{abstract}

\section{Introduction}

In recent years six-dimensional compact manifolds with $S U(3)$ structure have become a serious option for compactifications of string theories. We have been led to consider $S U(3)$-manifolds more general than Calabi-Yau (CY) manifolds both from theoretical and phenomenological considerations.

An $S U(3)$ manifold can be characterized by a geometric quantity known as the intrinsic torsion, which measures the deviation of the holonomy group of the Levi-Civita connection $\nabla^{6}$ from $S U(3)$ 2. An interesting subclass of $S U(3)$-manifolds are the half-flat (HF) manifolds. On a HF manifold half of the possible components of the intrinsic torsion vanish (see 2 for more details). Certain HF manifolds, dubbed as HF mirror manifolds in [3], arise naturally from considerations of mirror symmetry in the presence of

*Tibra_Ali@baylor.edu

†Gerald_Cleaver@baylor.edu
NS-NS background fluxes in type II string theories [4.

Although originating from type II theories these conjectured HF mirror manifolds are natural compactification spaces for the $E_{8} \times E_{8}$ heterotic string theory for two reasons. First, they are considered to be 'small' deformations of CY's. Thus one expects the same spectrum of low-energy effective fields from them as one gets from CY compactifications. Indeed, implicit in the work of [4] is the fact that the Euler characteristic of a HF mirror manifold is the same as the Euler characteristic of the 'underlying' CY manifold.

The second reason for compactifying the heterotic string on HF mirror manifolds has to do with modulistabilization. Heterotic strings suffer from the apparent drawback that they offer fewer 'fluxes' to be turned-on. On HF manifolds fluxes are geometrically encoded. In fact HF manifolds may be thought of as CY manifolds on which the Ricci-curvature has gained non-zero vacuum expectation value 11 Thus to include some of the desirable features of flux compactifications [6, 7] in the heterotic setting it is natural to turn to HF manifolds.

The study of heterotic string theory on HF mirror manifolds was initiated in [3] and in a recent communication [1] the configuration of the background gauge field was explored. In [1] it has been noted that although the holonomy group of the Levi-Civita connection on HF manifolds is not $S U(3)$, a version of standard embedding still leads to the breakdown of $E_{8} \times E_{8}$ to $E_{6} \times E_{8}$ as in the case of CY compactifications [8]. In reaching this conclusion (which reverses a previous assertion [3] that $E_{8} \times E_{8}$ breaks down to $\left.S O(10) \times E_{8}\right)$ the authors of [1] have made use of the 'adiabatic principle' as well as the conjec-

\footnotetext{
${ }^{1}$ This point of view regarding the relationship between HF and CY manifolds is currently under investigation [5].
} 
tured 'Gukov' superpotential. Although conjectures are essential to the progress of string theory this is a case in which there seems to be a much more direct and transparent route leading to the same conclusion. This is the subject of this note.

Our conclusions are essentially the product of two results: Hitchin's theorem about the relationship between $G_{2}$-holonomy cylinders and $\mathrm{HF}$ manifolds $[9$, and Hull's observations 10 regarding the nature of ambiguity in the Green-Schwarz anomaly cancellation condition.

In the next section we present our argument, relegating a technicality to an appendix.

\section{The Standard Embedding on Half-Flat Manifolds}

We start by asking the following question: What is the ten-dimensional space-time whose low-energy dynamics is described by the effective action of $E_{8} \times E_{8}$ heterotic string theory on HF manifolds? In the type II context supergravity no-go theorems forbid $\mathbb{R}^{1,3}$ as a solution to the effective theory. In fact the BPS solution turns out to be a domain wall. This solution can be taken over to the heterotic setting easily with the adjustment of the standard embedding described below. Thus it is clear that the ten-dimensional solution that is relevant for the case at hand is the lift of the domain wall which is the direct product spacetime $\mathbb{R}^{1,2} \times \mathcal{Z}_{7}$ where $\mathcal{Z}_{7}$ is a $G_{2}$-holonomy generalized cylinder 2 with the natural metric

$$
\begin{aligned}
d s^{2} & =g_{M N} d x^{M} d x^{N} \\
& =d z^{2}+g(z, y)_{m n} d y^{m} d y^{n}
\end{aligned}
$$

where $x^{M}$ with $M=1, \ldots, 6, z$ are coordinates on $\mathcal{Z}_{7}$ and $y^{m}$ with $m=1, \ldots, 6$ are coordinates on six dimensional hypersurfaces ${ }^{z} \mathcal{M}_{6}$ with the metric $g(z, y)_{m n}$ for a fixed value of $z$. Then according to Hitchin 9 ${ }^{z} \mathcal{M}_{6}$ are HF manifolds.

With the dilaton constant in $\mathbb{R}^{1,2} \times \mathcal{Z}_{7}$ background it is easy to see that the gravitino variation condition is satisfied. On $\mathcal{Z}_{7}$ we have a Majorana spinor $\epsilon$ satisfying

$$
\nabla_{M}^{7} \epsilon=0
$$

where $\nabla^{7}$ is the Levi-Civita connection on $\mathcal{Z}_{7}$. The integrability condition for the above equation is

$$
R_{M N P Q}^{7} \Gamma^{P Q} \epsilon=0 .
$$

\footnotetext{
2The term 'generalized cylinder' was introduced in 11 to describe space-times whose metrics have the form of eq.(1).
}

where $\Gamma^{M N}$ is the antisymmetrized product of two Dirac matrices on $\mathcal{Z}_{7}$. To solve the gaugino supersymmetry condition we adopt

$$
A=\omega^{7}
$$

where $A$ is the $E_{8} \times E_{8}$ Yang-Mills gauge potential and $\omega^{7}$ is the spin-connection with $G_{2}$ holonomy that corresponds to the Levi-Civita connection $\nabla^{7}$. This is the standard embedding for $G_{2}$ holonomy background. Because of (3) we then have

$$
F_{M N} \Gamma^{M N} \epsilon=0
$$

The remaining condition, coming from the vanishing of the dilatino variation, is then satisfied, with constant dilaton, by choosing $H_{3}=0$, where $H_{3}$ is given by

$$
H_{3}=d B_{2}+\mathcal{O}\left(\omega^{7}\right)-\mathcal{O}(A)
$$

with $\mathcal{O}\left(\omega^{7}\right)$ and $\mathcal{O}(A)$ being the Chern-Simons 3forms in the Lorentz and the gauge sectors, respectively. The choice eq. (4) then leads to the GreenSchwarz anomaly cancellation condition

$$
d H_{3}=\operatorname{tr} R \wedge R-\frac{1}{30} \operatorname{Tr} F \wedge F
$$

being satisfied with $H_{3}=0$. What we have outlined above is a special case of an 'instanton' solution explored in more detail in [12. If we were interested in the three dimensional effective action of the compactification on a (say, compact) $G_{2}$ holonomy manifold then the above construction would lead $E_{8} \times E_{8}$ to break down to $F_{4} \times E_{8}$. This would be consistent with the fact that there is no notion of chirality in three dimensions (since $F_{4}$ doesn't lead to chiral multiplets). However, we are interested in knowing what is the condition that descends on the HF slices ${ }^{z} \mathcal{M}_{6}$ from the above condition.

Since HF manifolds are manifolds with $S U(3)$ structure there must exist a connection $\widetilde{\nabla}^{6}$ on this manifold with respect to which the globally defined Majorana spinor $\epsilon^{\prime}$, which implicitly defines the $S U(3)$ structure, is covariantly constant:

$$
\widetilde{\nabla}_{m}^{6} \epsilon^{\prime}=0
$$

However this connection is not the Levi-Civita connection $\nabla^{6}$. It differs from $\nabla^{6}$ by the intrinsic torsion whose detailed form is given in the appendix. The holonomy of the torsionful connection $\widetilde{\nabla}^{6}$ is still $S U(3)$ as can be seen from the integrability condition of (8)

$$
\widetilde{R}_{m n p q}^{6} \Gamma^{p q} \epsilon^{\prime}=0
$$


In the above equation the torsion term drops out due to (8). The globally defined spinor $\epsilon^{\prime}$ that defines the $S U(3)$ structure is the same spinor whose constancy in seven-dimensions leads to $G_{2}$ holonomy, i.e.

$$
\epsilon^{\prime}=\epsilon
$$

In the appendix we give an explicit demonstration of this fact using the explicit formulae, developed in [13, relating the intrinsic torsion of ${ }^{z} \mathcal{M}_{6}$ and the extrinsic curvature of the embedding of ${ }^{z} \mathcal{M}_{6}$ in $\mathcal{Z}_{7}$.

The above discussion strongly suggests that the natural embedding condition to choose on a HF manifold is then

$$
A=\widetilde{\omega}^{6}
$$

where $\widetilde{\omega}^{6}$ is the metric-compatible torsionful spinconnection whose holonomy is $S U(3)$. The GreenSchwarz anomaly cancellation condition is then no longer (7) but is instead give by

$$
d \widetilde{H}_{3}=\operatorname{tr} \widetilde{R}^{6} \wedge \widetilde{R}^{6}-\frac{1}{30} \operatorname{Tr} F \wedge F
$$

with $\widetilde{R}^{6}$ being the same curvature that appeared in (9). We also have

$$
\widetilde{H}_{3}=d B_{2}+\mathcal{O}\left(\widetilde{\omega}^{6}\right)-\mathcal{O}(A)
$$

It was pointed out a while ago by Hull [10] that the anomaly in a gauge theory is always ambiguous up to a change in the connection by a tensor quantity. Changing to a new connection in the action (and hence the path-integral) simply implies a corresponding change in the counter-term that is needed to cancel the anomaly. This 'new' anomaly cancellation condition (12) is then solved by $\widetilde{H}_{3}=0$ and (11). Equation (11) is the standard embedding on $\mathrm{HF}$ manifolds. It implies $E_{8} \times E_{8}$ breaks down to $E_{6} \times E_{8}$ just as in the case of $C Y$ compactifications.

\section{Conclusions and Outlook}

In this paper we have argued that the ten dimensional solution that corresponds to the compactification of $E_{8} \times E_{8}$ heterotic string theory on a half-flat manifold is the direct product space-time $\mathbb{R}^{1,2} \times \mathcal{Z}_{7}$ where $\mathcal{Z}_{7}$ is a generalized cylinder with $G_{2}$ holonomy à la Hitchin [9]. The supersymmetry conditions and Green-Schwarz anomaly cancellation condition are then satisfied by embedding the Levi-Civita connection of $\mathcal{Z}_{7}$ in the gauge connection. This implies a standard embedding on the half-flat slices which is given by (11) which lead to the breakdown of $E_{8} \times E_{8}$ to $E_{6} \times E_{8}$. However, this implies that the natural variables for HF manifolds are not any of the standard formulations of heterotic string theory. But the existence of such a formulation is guaranteed by an ambiguity in the Green-Schwarz anomaly [10].

It is important to note that, unlike in [1, we did not assume that the HF manifold had to be some sort of 'small' or 'adiabatic' deformation of an underlying CY. Once the ansatz (11) is adopted the anomaly cancellation condition (12) is satisfied exactly. Our results are valid for any half-flat manifold (including Nilmanifolds). However, for phenomenological purposes one is mainly interested in HF mirror manifolds. It is our hope that the fact the approach taken here is independent of some of the approximations made in [1] puts their effort on firmer ground.

Since the full ten dimensional solution breaks down to $F_{4} \times E_{8}$, it is a prediction of our work that the domain wall solutions of the effective theory on HF manifolds will spontaneously break $E_{6}$ down to $F_{4}$. In passing we note that the decomposition of the 'visible' $E_{8}$ in terms of $F_{4}$ and $G_{2}$, which naturally comes out of HF manifolds, is reminiscent of the group-structure of the unification scenario recently proposed by Lisi [14.

Because our conclusions are based on a bona fide ten-dimensional solution one cannot a priori comment on the standard embedding on $S U(3)$ manifolds more general than HF manifolds. In our view one needs to first look at the ten-dimensional solutions before making ansätze for dimensional reductions on six dimensional manifolds with arbitrary $S U(3)$-structures.

Since the ten-dimensional effective actions for heterotic string theories contain higher order curvature terms it is not immediately clear that, despite the standard embedding, all the curvature dependent terms are computable on HF mirror manifolds. However, since the Ricci-curvature for HF manifolds have now been computed in terms of variables familiar from CY compactifications [13] it seems not unlikely that one can compute the effective action in these new variables. This effective action would be a consistency check on the one derived in [1] and would perhaps be related to the latter action by a change of variables. This and other issues are now under investigation [5].

\section{Acknowledgment}

It is a pleasure to thank S. Majhi for insightful and stimulating discussions. 


\section{Appendix: $S U(3)$ holonomy and $G_{2}$ holonomy}

We are interested in the six-dimensional condition that descends from the seven-dimensional standard embedding that we discussed above. Hitchin's theorem 9] implies that the relevant connection in six dimensions is the one with torsion. In this section we verify this explicitly. For more details on the origin of the following formulae the reader is referred to [13.

An $S U(3)$ manifold admits a globally defined two form $J$ (related to an almost complex structure $J_{m}{ }^{n}$ ) and a complex three form $\Omega=\Omega^{+}+i \Omega^{-}$which is of type $(3,0)$ with respect to $J_{m}{ }^{n}$. On a Calabi-Yau manifold both of these forms are closed. On a halfflat manifold one has instead

$$
\begin{aligned}
J \wedge d J & =0 \\
d \Omega^{-} & =0
\end{aligned}
$$

To show (10) we start with the equation (2) and look at the components lying along the half-flat slices:

$$
\nabla_{m}^{7} \epsilon=0 .
$$

where $m=1, \ldots, 6$. Let us denote by $\nabla^{6}$ the Levi-Civita connection in six-dimensions. The GaußWeingarten equation then gives [13]:

$$
\nabla_{m}^{7} \epsilon=\nabla_{m}^{6} \epsilon+\frac{1}{2} K_{m}^{n} \Gamma_{n} \Gamma^{7} \epsilon .
$$

Where $K_{m n}$ is the second fundamental form of the embedding of the half-flat manifold in a $G_{2}$ holonomy cylinder. In 13 it was shown that for half-flat manifolds the intrinsic contorsion $\kappa_{r s t}$ (which is equivalent to the intrinsic torsion) is related to $K_{m n}$ via

$$
\kappa_{r s t}=\frac{1}{2} K_{r}^{m} \Omega_{m s t}^{+}
$$

and its inverse

$$
K_{r}^{p}=\frac{1}{2} \Omega^{+p s t} \kappa_{r s t} .
$$

Then (16) becomes

$$
\nabla_{m}^{7} \epsilon=\nabla_{m}^{6} \epsilon+\frac{1}{4} \Omega^{+n s t} \kappa_{m s t} \Gamma_{n} \Gamma^{7} \epsilon .
$$

We shall now show the connection on the right hand side (and hence the truncated connection on the left hand side) has $S U(3)$ holonomy. Let us first recall the following Clifford algebra identities:

$$
\begin{aligned}
& \Gamma_{m n p}=\Gamma_{m n} \Gamma_{p}+g_{p m} \Gamma_{n}-g_{p n} \Gamma_{m} \\
& \Gamma_{m n p}=\Gamma_{m} \Gamma_{n p}+g_{p m} \Gamma_{n}-g_{m n} \Gamma_{p} .
\end{aligned}
$$

We also need the following Fierz identity, which is simply a statement of the decomposition of identity on the spinorial vector space in six spatial dimensions:

$$
\mathbf{1}_{8 \times 8}=\epsilon \bar{\epsilon}+\Gamma_{7} \epsilon \bar{\epsilon} \Gamma^{7}+\Gamma_{m} \epsilon \bar{\epsilon} \Gamma^{m}
$$

We can now consider

$$
\begin{aligned}
\Gamma_{m n} \Gamma^{7} \epsilon & =\left\{\epsilon \bar{\epsilon}+\Gamma_{7} \epsilon \bar{\epsilon} \Gamma^{7}+\Gamma_{r} \epsilon \bar{\epsilon} \Gamma^{r}\right\} \Gamma_{m n} \Gamma^{7} \epsilon \\
& =\epsilon \bar{\epsilon} \Gamma_{m n} \Gamma^{7} \epsilon+\Gamma_{r} \epsilon \bar{\epsilon} \Gamma^{r} \Gamma_{m n} \Gamma^{7} \epsilon
\end{aligned}
$$

where in going from the first line to the second we have used the fact that for any commuting Majorana spinor $\epsilon$ in six dimensions we have

$$
\bar{\epsilon} \Gamma_{7} \Gamma_{m n} \Gamma^{7} \epsilon=\bar{\epsilon} \Gamma_{m n} \epsilon=0 .
$$

Next we use the following definitions [13]

$$
\begin{aligned}
J_{m n} & =i \bar{\epsilon} \Gamma_{7} \Gamma_{m n} \epsilon \\
\Omega_{m n p}^{+} & =\bar{\epsilon} \Gamma_{m n p} \Gamma_{7} \epsilon
\end{aligned}
$$

in the identity (23) and obtain, after multiplying through $\Gamma^{7}$,

$$
\Gamma_{m n} \epsilon=-i J_{m n} \Gamma^{7} \epsilon-\Omega_{r m n}^{+} \Gamma^{r} \Gamma^{7} \epsilon .
$$

Using this in (19) we get

$$
\nabla_{m}^{7} \epsilon=\nabla_{m}^{6} \epsilon+\frac{1}{4} \kappa_{m s t}\left\{-i J^{s t} \Gamma^{7}-\Gamma^{s t}\right\} \epsilon .
$$

In 2] it was shown that the torsion or the contorsion on an $S U(3)$ manifold decomposes into five $S U(3)$ modules. On a half-flat manifold some of these modules survive which we denote by $W_{1}^{+}, W_{2}^{+}$and $W_{3}$. More details on these modules relevant for the present computation can be found in 13 . Here we note that $W_{1}^{+}$is a real scalar, $W_{2}^{+}$is a real, primitive (1,1)-type 2 -form and $W^{3}$ is a complex, primitive (2,1)-type 3 form. Now it is clear from the following expression of the contorsion tensor on a half-flat manifold 13

$$
\begin{aligned}
\kappa_{s r t} & =\frac{1}{4} W_{1}^{+} \Omega_{s r t}^{+}+\frac{1}{4}\left(W_{2}^{+}\right)_{s q} \Omega_{r t}^{-q} \\
& +\frac{i}{2}\left\{\left(W_{3}\right)_{s u v} \Pi_{r}^{+u} \Pi_{t}^{+v}-\left(\bar{W}_{3}\right)_{s u v} \Pi_{r}^{-u} \Pi_{t}^{-v}\right\},
\end{aligned}
$$

where

$$
\left(\Pi^{ \pm}\right)_{p}{ }^{q}=\frac{1}{2}\left[\delta_{p}^{q} \mp i J_{p}^{q}\right],
$$

that

$$
J^{r t} \kappa_{\text {srt }}=0 .
$$

Thus (27) becomes

$$
\begin{aligned}
\nabla_{m}^{7} \epsilon & =\nabla_{m}^{6} \epsilon-\frac{1}{4} \kappa_{m s t} \Gamma^{s t} \epsilon \\
& \equiv \widetilde{\nabla}_{m}^{6} \epsilon .
\end{aligned}
$$


As a consequence of (2) we have for the connection $\widetilde{\nabla}$ with contorsion $\kappa$

$$
\widetilde{\nabla}_{m}^{6} \epsilon=0
$$

which means that the holonomy of $\widetilde{\nabla}^{6}$ is $S U(3)$. Hence $\epsilon=\epsilon^{\prime}$ where the latter is the globally defined spinor on the HF manifold which determines its $S U(3)$ structure.

\section{References}

[1] S. Gurrieri, A. Lukas and A. Micu, (2007), arXiv:0709.1932 [hep-th].

[2] S. Chiossi and S. Salamon, Differential geometry, Valencia, 2001 (2001) 115, math-dg/0202282.

[3] S. Gurrieri, A. Lukas and A. Micu, Phys. Rev. D70 (2004) 126009, hep-th/0408121.

[4] S. Gurrieri et al., Nucl. Phys. B654 (2003) 61, hep-th/0211102.

[5] T. Ali and G.B. Cleaver, In preparation.

[6] M. Grana, Phys. Rept. 423 (2006) 91, hepth/0509003.

[7] M.R. Douglas and S. Kachru, Rev. Mod. Phys. 79 (2007) 733, hep-th/0610102.

[8] P. Candelas et al., Nucl. Phys. B258 (1985) 46.

[9] N.J. Hitchin, (2001), math/0107101.

[10] C.M. Hull, Phys. Lett. B167 (1986) 51.

[11] C. Bär, P. Gauduchon and A. Moroianu, Math. Zeitschr. 249 (2005) 545.

[12] P. Ivanov and S. Ivanov, Commun. Math. Phys. 259 (2005) 79, math/0312094.

[13] T. Ali and G.B. Cleaver, JHEP 05 (2007) 009, hep-th/0612171.

[14] A.G. Lisi, (2007), arXiv:0711.0770 [hep-th]. 